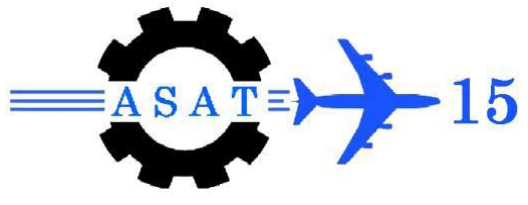

\title{
On the Study of Compact and Multi-Band CRLH Meta-Material Antenna for Wireless Applications
}

\author{
M. A. Abdalla ${ }^{*}$, A. A. Ibrahim ${ }^{\dagger}$, A. B. Abdel-Rahman ${ }^{\ddagger}$ and H. F. A. Hamed
}

\begin{abstract}
This paper presents a design of a small size composite right-left handed metamaterial multi-band antenna for wireless applications. The proposed antenna consists of two left handed cells. The proposed antenna operates at different frequency bands. The proposed antenna has compact size (its size is only 38 X $22 \mathrm{~mm} 2$ ). The design represents size reduction of approximately $65 \%$ compared to conventional patch antennas operating at the same frequencies. The size reduction and low frequency ratios have been achieved due to the nonlinearity behavior of the left-handed region of composite right-left handed transmission line.
\end{abstract}

Keywords: CRLH, left handed, meta-material, multi -band antenna

\section{Introduction}

The repaid growth of wireless communication leads to a greet demand in designing antennas with multi-band, high performance and easy to combine with other devices [1].Multi-band antennas are very useful for wireless applications due to only one antenna can cover several frequency bands. Several methods for obtaining multi-band characteristics have been developed. The multi-band operations are achieved by adopting multi-branched strips [2-4] and adding parasitic elements [5-8] to resonate at different frequencies in a single radiating device. However, these solutions generally suffer from certain drawbacks, including a large volume, a large ground plane, and a large frequency ratio. One possible solution to realize compact multi-band antennas with low frequency ratios could be utilization of metamaterial cells. Left handed (LH) transmission line (TL) is characterized by anti-parallel phase and group velocities. Also, it has a nonlinear progressive phase [9]-[11]. Because of these properties, compact microwave devices such as power dividers, filters, phase shifter, resonators, and antennas have been introduced [12]-[14]. Compact and multi bands antennas including novel zero ${ }^{\text {th }}$ order resonator antenna (ZORA) are presented for different wireless applications [15]-[16].

In this paper, we introduce a design of CRLH (TL) metamaterial multi-band antenna. The proposed metamaterial antenna was designed employing only two cells of left handed transmission line. The left handed metamaterial consists of interdigital capacitor and short circuit stub work as inductor. The proposed antenna has compact size .The size reduction and low frequency ratios have been achieved due to the nonlinearity behavior of the left-handed region of CRLH(TL). The theoretical concepts of the designed antenna are introduced. The designed results have been validated using the electromagnetic full wave simulations.

\footnotetext{
*Egyptian Armed Forces, Egypt; maaabdalla@ieee.org

† Faculty of Engineering, El-Minia University, engahmedabdel_monem@yahoo.com

* E-Just University - Alexandria, Egypt, adel_b15@yahoo.com 


\section{Theory}

The explanation of TL approach of LHM as an ideal LHTL is not accurate so, the realization of LHM comprises both LH contribution due to the elements effect and RH contribution due to the parasitic series inductance and shunt capacitance effect of TL elements. Because of this contribution a practical TL approach LHM is referred as a composite right / left handed (CRLH) TL. The equivalent circuit approach of a lossless CRLH unit cell can be introduced as shown in Fig. 1.

In the equivalent circuit model, RH parameters consist of the series inductor and the shunt capacitor of the hosting TL while the series capacitor and shunt inductor are corresponding to the loading LH elements. It is seen that, at lower frequency, the RH elements, CR and LR, will be open circuited and short circuited respectively. Thus, the CRLH unit cell equivalent circuit reduces to that of LH equivalent circuit and hence it acts as a nearly ideal LH TL. Similarly, at higher frequency, the CRLH unit cell equivalent circuit reduces to that of RH equivalent circuit and hence it acts as an ideal RH TL.

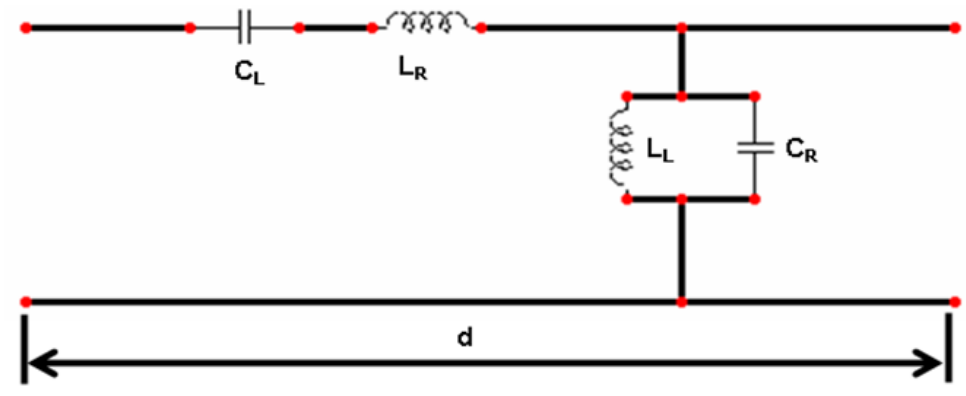

Fig. 1 The equivalent circuit model of CRLH transmission line.

The basic concept of transmission line resonating antenna is based on achieving resonance at frequencies which fulfill the full wave conditions as

$$
\beta l=n \pi
$$

where 1 is the total physical length of the resonating antenna, $\mathrm{n}$ is an integer specifies the resonating order and $\beta$ is the guided propagation constants within the antenna substrate. In conventional microstrip patch antenna, the constant integer $\mathrm{n}$ is always positive. In conventional transmission line, the propagation constant is directly proportional to frequency; changes linearly with frequency. Thus, the operating condition for resonant antenna can be satisfied only at the harmonic frequencies of the antenna. On the other hand, the propagation constant is varying with the frequency in non linear way. Therefore, it can be implemented to introduce arbitrary operating multiband which is not essentially to be multiple of the fundamental frequency harmonic.

Also, by cascading metamaterial transmission line with conventional transmission line, the propagation constant can be arbitrary positive or negative or even zero. This can be illustrated in Fig.2. Hence, the radiation condition is

$$
\beta l=\beta_{l} d_{l}+\beta_{r} d_{r}=n \pi
$$

where $\beta_{1}$ and $\beta_{\mathrm{r}}$ are the propagation constants of LH and RH sections, respectively, where $\mathrm{d}_{1}$ and $d_{r}$ represent the length of the sections, respectively. In this case, the integer can be expressed as

$$
n=0, \pm 1, \pm 2, \pm 3, \ldots \ldots \ldots \ldots . \pm(N-1)
$$




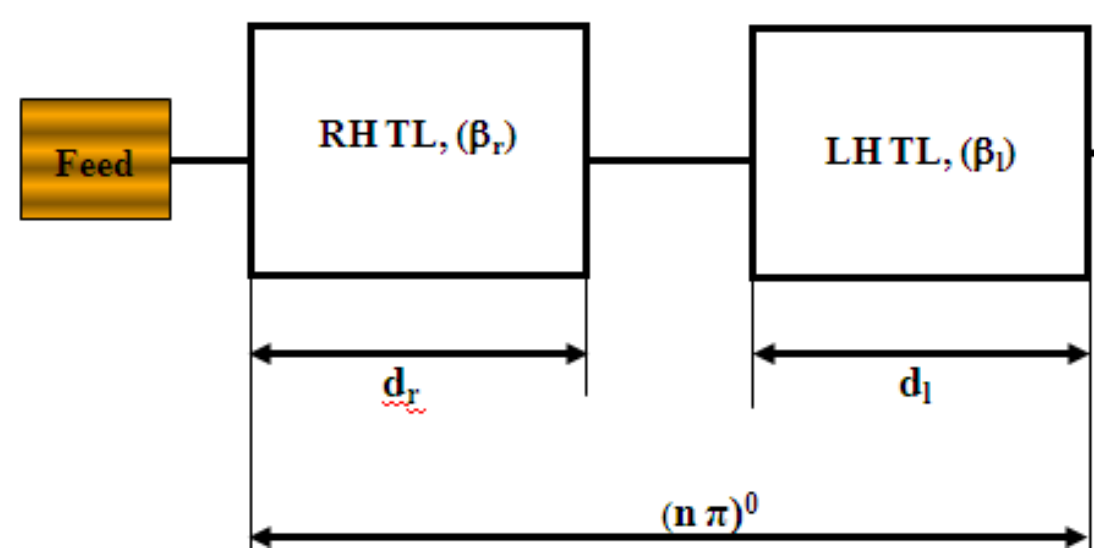

Fig. 2 The equivalent block diagram of metamaterial left handed antenna

The left handed and right handed propagation constants are determined according to the loading elements and parasitic elements [11] which can be expressed as

$$
\begin{aligned}
& \beta_{l}=\left(\frac{-1}{\omega \sqrt{C_{L}} L_{L}}\right) \\
& \beta_{r}=\left(\omega \sqrt{C_{R} L_{R}}\right)
\end{aligned}
$$

A practical LH unit cell contains a RH patristic effect which can do the theoretical cascading LH and RH sections. Hence, the phase shift of a CRLH TL can be extracted from the phase of the transmission coefficient S21 as follow in (6) as [11];

$$
\begin{aligned}
& \varphi_{C R L H}=\tan ^{-1}\left(\frac{\left(\left(\omega / \omega_{s e}\right)^{2}-1\right)(1-\chi / 4) / C_{L} Z_{C R L H}\left(\left(\omega / \omega_{s h}\right)^{2}-1\right) Z_{C R L H} / L_{L}}{\omega(2-\chi)}\right) \\
& \chi=-\left(\frac{\omega}{\omega_{R}}\right)^{2}+\left(\frac{\omega_{L}}{\omega}\right)^{2}-\left(\frac{C_{R}}{C_{L}}+\frac{L_{R}}{L_{L}}\right) \\
& \omega_{R}=\frac{1}{\sqrt{L_{R} C_{R}}} \\
& \omega_{L}=\frac{1}{\sqrt{L_{L}} C_{L}} \\
& \omega_{s h}=\frac{1}{\sqrt{L_{L} C_{R}}} \\
& \omega_{s e}=\frac{1}{\sqrt{L_{R} C_{L}}} \\
& Z_{C R L H}=Z_{L} \sqrt{\frac{\left(\omega / \omega_{s e}\right)^{2}-1}{\left(\omega / \omega_{s h}\right)^{2}-1}}=Z_{R} \sqrt{\frac{\left(\omega_{s e} / \omega\right)^{2}-1}{\left(\omega_{s h} / \omega\right)^{2}-1}}
\end{aligned}
$$




\section{Antenna Design Procedures}

The layout of single element ZORA metamaterial antenna is shown in Fig. 3. The antenna was designed using one $\mathrm{LH}$ cell connected to a $50 \Omega \mathrm{RH}$ feeding line. The antenna consists of a series interdigital capacitor and a shunt stub inductor. The antenna was designed on substrate FR4 with relative permittivity $=4.4$, a dielectric loss tangent $=0.025$ and thickness $(\mathrm{h})$ $=1.6 \mathrm{~mm}$. The operation frequency of this antenna is $2.1 \mathrm{GHz}$ for UMTS application.

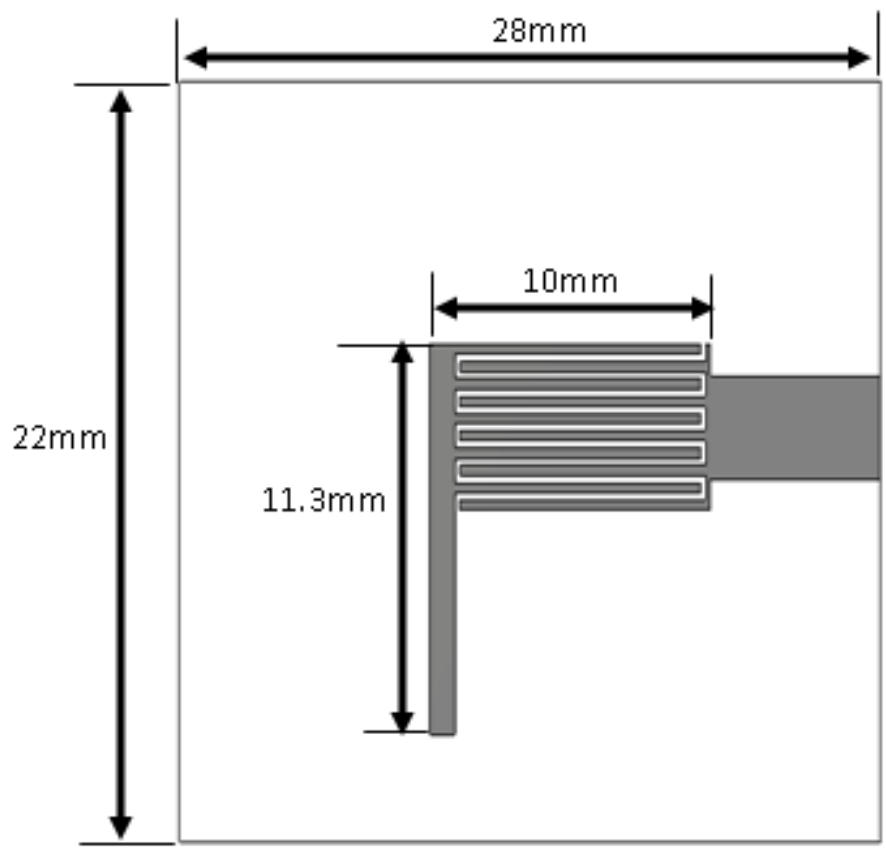

Fig. 3 2D layout of single element CRLH antenna

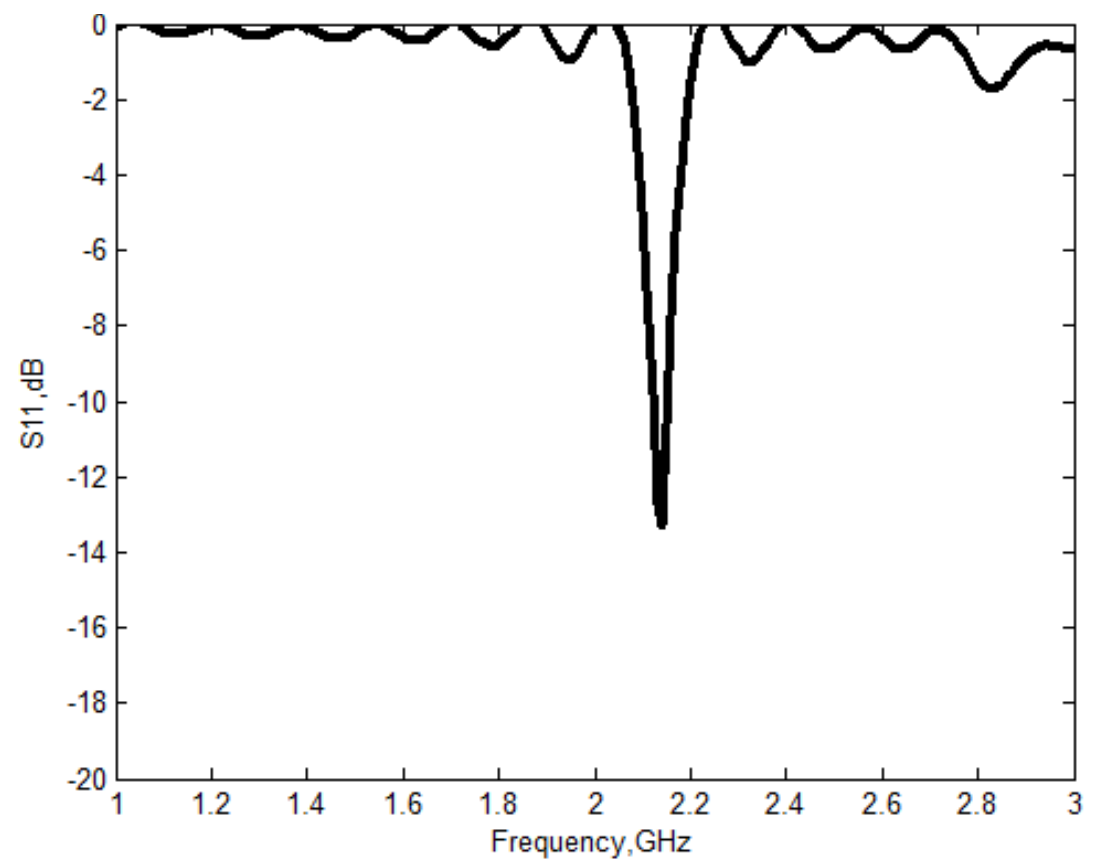

Fig. 4 Simulated return loss of single element CRLH antenna 
The operating frequency of the resonant antenna was designed to fulfill the zero ${ }^{\text {th }}$ order resonance (indicated in (1) for $n=0$ ). The antenna has compact size (its size is only $28 \times 22$ $\mathrm{mm} 2$ ) as compared with conventional antenna works at the same frequency. By optimizing the dimensions of the unit cell, a zero phase shift can be obtained at $2.1 \mathrm{GHz}$. The simulated return loss of single element metamaterial antenna is shown in Fig. 4. As shown in the figure, the antenna can achieve better than $-14 \mathrm{~dB}$ reflection coefficient at $2.1 \mathrm{GHz}$. For more confirmation about the single element metamaterial antenna initial design, its simulated radiation pattern in both $\mathrm{E}(\mathrm{XZ}$ plane with $\Phi=0)$ and $\mathrm{H}$ plane (YZ plane with $\Phi=90)$ are shown in Fig.5 respectively. It is obvious that the patterns are very comparable of typical pattern of conventional microstrip patch antenna [17].

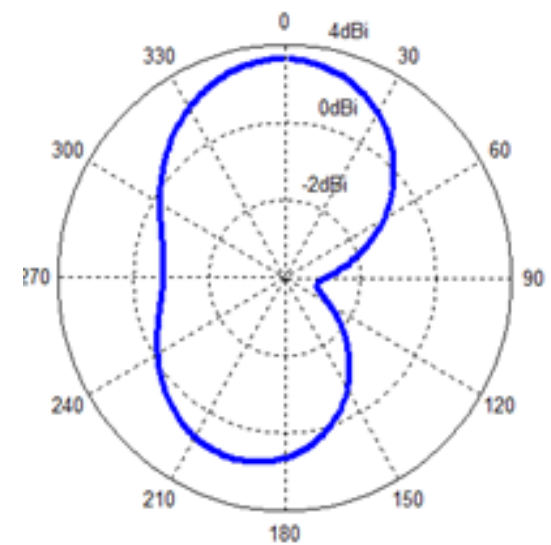

(a)

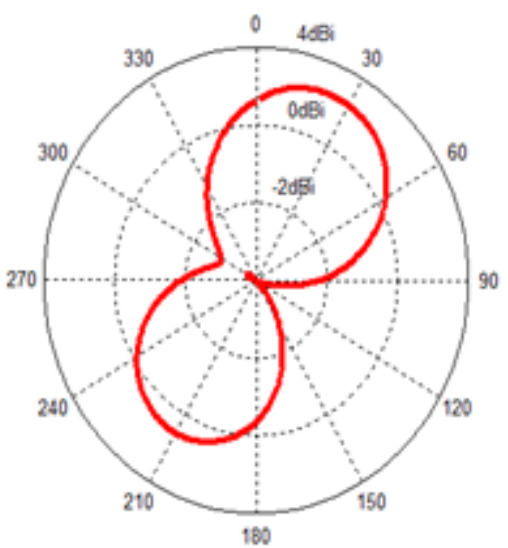

(b)

Fig. 5 The Simulated field pattern of single element CRLH antenna (a) E and (b) H Plane at 2.1GHz

\section{Proposed Multi-Band Antenna}

The basic structure of the proposed antenna is shown in Fig.6. The antenna was designed using two LH cell connected to a $50 \Omega \mathrm{RH}$ feeding line. Referring to (3) the number of $\mathrm{n}$ is equal to three 0,1 and -1 because of increasing the number of cells. So, the proposed antenna behaved like multi-band antenna. The antenna was designed on substrate FR4 with relative permittivity $=4.4$, a dielectric loss tangent $=0.025$ and thickness $(\mathrm{h})=1.6 \mathrm{~mm}$.

The simulated return loss of two elements metamaterial antenna is shown in Fig. 7. As shown in the figure, the antenna operates at triple frequency bands better than $-12 \mathrm{~dB}$ and lower than $-9 \mathrm{~dB}$ for certain frequency of $3.65 \mathrm{GHz}$. The antenna operates at $2.4 \mathrm{GHz}$ for Bluetooth and wireless LAN applications, $3 \mathrm{GHz}$ for security and surveillance applications and $4.3 \mathrm{GHz}$ for WIMAX applications. The antenna has compact size (its size is only $38 \mathrm{X} 22 \mathrm{~mm} 2$ ) as compared with conventional antenna works at the same frequency. For more confirmation about the proposed multi band antenna, its simulated radiation pattern in both $\mathrm{E}$ (XZ plane with $\Phi=0$ ) and $\mathrm{H}$ plane (YZ plane with $\Phi=90$ ) are shown in Fig.8, Fig9and Fig.10. It is obvious that the directivity of this antenna at $2.4 \mathrm{GHz}$ is $3.5 \mathrm{dBi}, 5 \mathrm{dBi}$ at $3 \mathrm{GHz}$ and $4.9 \mathrm{dBi}$ at $4.3 \mathrm{GHz}$. 


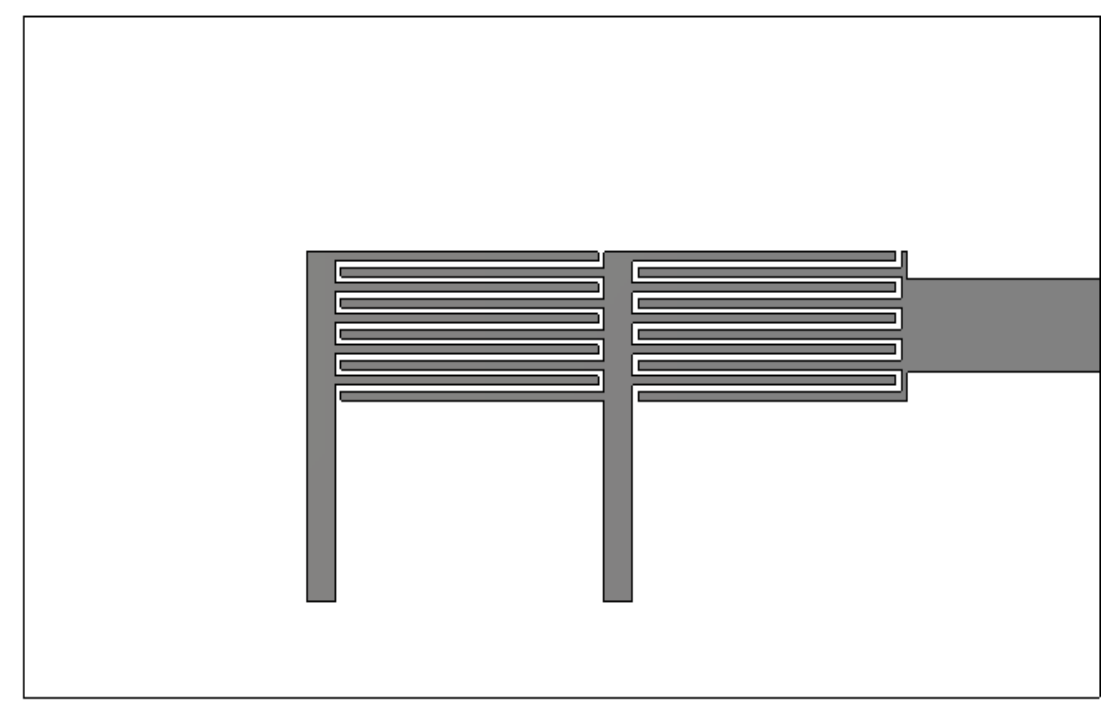

Fig. 6 2D layout of proposed Multi-band antenna

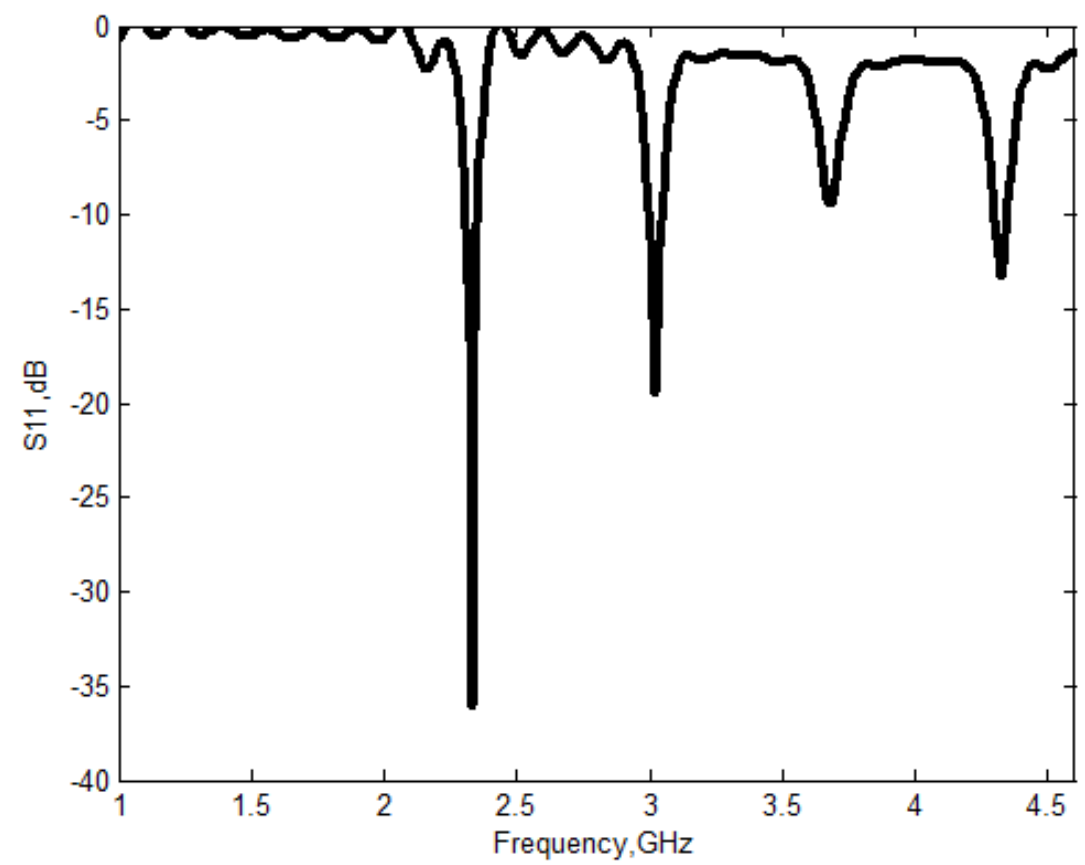

Fig. 7 Simulated return loss of proposed multi -band antenna 


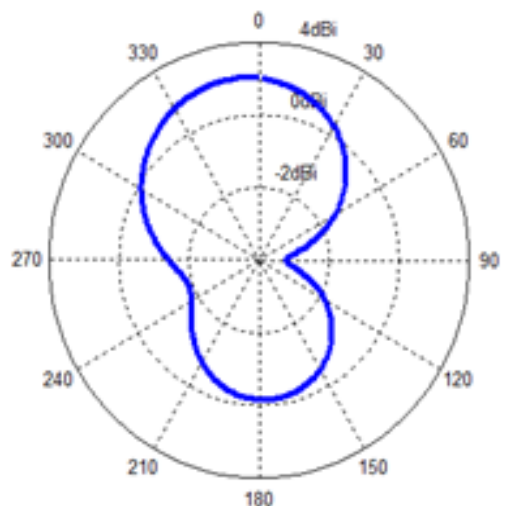

(a)

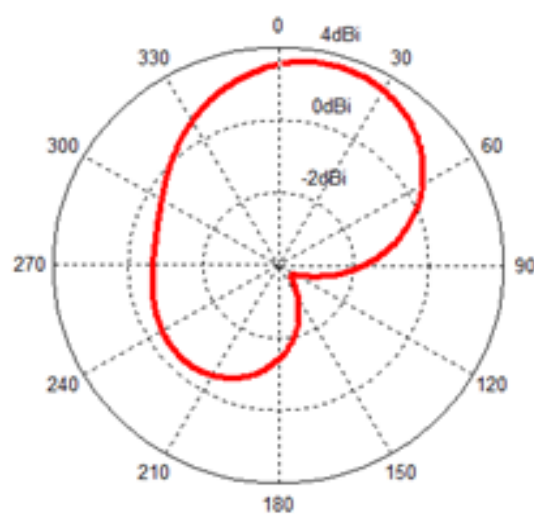

(b)

Fig. 8 The Simulated field pattern of proposed antenna

(a) E and (b) H Plane at $2.4 \mathrm{GHz}$

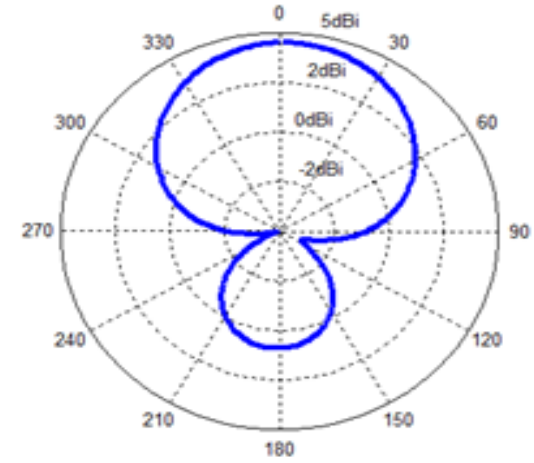

(a)

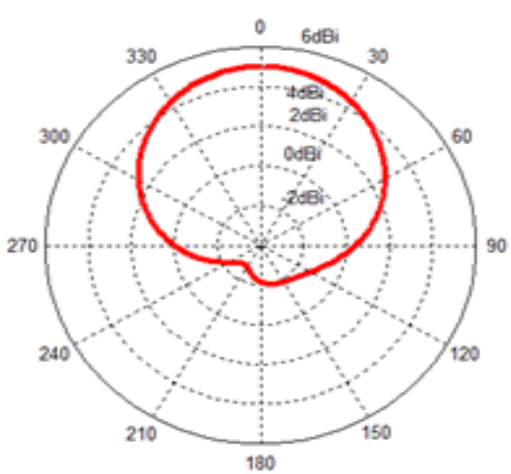

(b)

Fig. 9 The Simulated field pattern of proposed antenna (a) E and (b) H Plane at $3 \mathrm{GHz}$

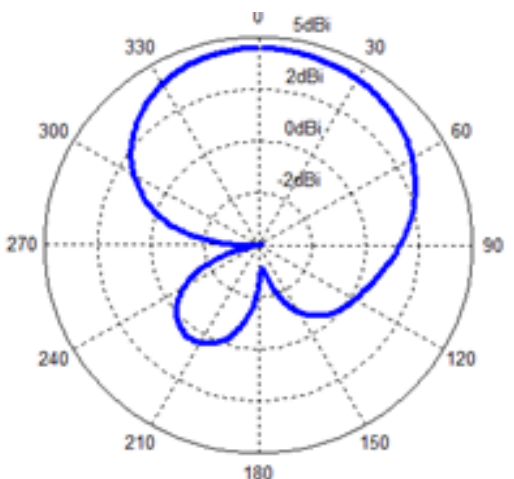

(a)

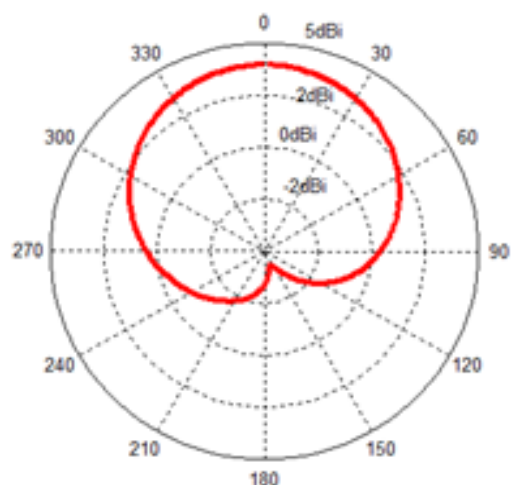

(b)

Fig. 10 The Simulated field pattern of proposed antenna

(a) E and (b) H Plane at 4.3GHz 


\section{Conclusion}

A design of a small size composite right-left handed meta-material multi-band antenna for wireless applications has been introduced. The proposed antenna consists of two left handed cells. The proposed antenna operates at different frequency bands. The design has been represented size reduction of approximately $65 \%$ compared to conventional patch antennas operating at the same frequencies. The size reduction and low frequency ratios have been achieved due to the nonlinearity behavior of the left-handed region of composite right-left handed transmission line dispersion diagram.

\section{References}

[1] Wong, K. L., Planar Antennas for Wireless Communications, John Wiley \& Sons, Inc., 2003.

[2] Song, K., Y. Z. Yin, and B. Chen, "Triple-band open L-slot antenna with a slit and a strip for WLAN/WiMAX applications," Progress In Electromagnetics Research Letters, Vol. 22, 139-146,2011.

[3] Hu, W., Y. Z. Yin, P. Fei, and X. Yang, " Compact triband square- slot antenna with symmetrical L-strips for WLAN/WiMAX applications," IEEE Antennas and Wireless Propagation Letters, Vol. 10, 462-465, 2011.

[4] Jaw, J.-L. and J.-K. Chen, "CPW-fed hook-shaped strip antenna for dual wideband operation," Journal of Electromagnetic Waves and Applications, Vol. 22, No. 13, 18091818, 2008.

[5] Heidari, A. A., M. Heyrani, and M. Nakhkash, " A dual-band circularly polarized stub loaded microstrip patch antenna for GPS applications," Progress In Electromagnetics Research, Vol. 92, 195-208, 2009.

[6] Wang, E., J. Zheng, and Y. Liu, "A novel dual-band patch an- tenna for WLAN communication," Progress In Electromagnetics Research C, Vol. 6, 93-102, 2009.

[7] Ciais, P., R. Staraj, G. Kossiavas, and C. Luxey, "Design of an internal quad-band antenna for mobile phones," IEEE Microwave and Wireless Components Letters, Vol. 14, 148-150, 2004.

[8] Risco, S., J. Anguera, A. Anduar, A. Perez, and C. Puente, "Coupled monopole antenna design for multiband handset devices," Microwave and Optical Technology Letters, Vol. 52, 359-364, 2010.

[9] A. A. Oliner, "A planar negative-refractive-index medium without resonant elements," in Proc. IMS Int. Microwave Symp., Philadelphia, USA, 2003, pp. 191-194.

[10] G. V. Eleftheriades, A. K. Iyer, and P. C. Kremer, "Planar negative refractive index media using periodically L-C loaded transmission lines," IEEE Transactions on Microwave Theory and Techniques, vol. 50, pp. 2702-2712, , Dec. 2002.

[11] C. Caloz and T. Itoh, "Transmission line approach of left-handed (LH) materials and microstrip implementation of an artificial LH transmission line," IEEE Transactions on Antennas and Propagation, vol. 52, pp. 1159-66,. Dec 2004.

[12] C. Caloz and T. Itoh, Electromagnetic Metamaterials Transmission Line Theory and Microwave Applications. New Jersey: John Wiey \& Sons, 2006.

[13] G. V. Eleftheriades and K. G. Balmain, Negative Refractive Metamaterials. New Jersey: John Wiey \& Sons, 2005.

[14] M. A. Abdalla and Z. Hu, " Compact Novel CPW Ferrite Coupled Line Circulator with Left-handed Power Divider/Combiner", 2011 European Microwave Week, EuMW2011, Digest, Manchester, UK, October 9-14 2011, pp. 794-707. 
[15] M. A. Abdalla and Z. Hu, " Compact and Tunable Metamaterial Antenna for Multi-band Wireless Communication applications", 2011 IEEE AP-S International Antenna and Propagation Symposium Digest, Jun. 3-8, 2011, Spokane, USA, pp/ 2951-2953

[16] M. A. Abdalla and Z. Hu, " Compact Dual Band Meta-material Antenna for Wireless Applications", 2012 Loughborough Antennas \& Propagation Conference, 12-13 November 2012, Loughborough, UK.

[17] Constantine A. Balanis, 'Antenna Theory, analysis and design', New Jersy, Wiley, 2005. 